\title{
Soliton Solution for the BBM and MRLW Equations by Cosine-function Method
}

\author{
Rajan Arora*, Anoop Kumar \\ DPT, IIT Roorkee, Saharanpur Campus, Saharanpur, U.P., 247001, India
}

\begin{abstract}
In this paper, we obtained a traveling wave solution by using cosine-function algorithm for nonlinear partial differential equations. Here, the method is used to obtain the exact solutions for two different types of nonlinear partial differential equations such as, Benjamin-Bona-Mahony (BBM) equation and Modified Regularized Long Wave (MRLW) equation which are the important soliton equations.
\end{abstract}

Keywords Cosine-Function Method, Benjamin-Bona-Mahony (BBM) Equation, Modified Regularized Long Wave (MRLW) Equation

\section{Introduction}

As we all know, nonlinear evolution equations (NEEs) are widely used to describe complex phenomena in various fields of science, such as fluid mechanics, plasma physics, solid state physics and optical fibres, etc. In order to better understand these nonlinear phenomena as well as further apply them in the practical life, it is important to seek their more exact solutions.

In recent year many power methods have been developed for finding the exact solution of nonlinear problems, such as tanh function method, modified extended tanh function method, sine-cosine method, inverse scattering transformation, Hirota bilinear method, Backlund transformation, Darboux transformation, homogeneous balance method, Jacobian elliptic function expansion method and its generalization, F-expansion method and so on[1-23]. Among them the Riccati equations method which was first presented by Conte et al in 1992 to seek more new solitary wave solutions to NEEs that can be expressed as polynomial in two elementary functions which satisfy a projective Riccati equation[14]. In[19], Huang and Zhang developed a variable-coefficient projective Riccati equation method for certain nonlinear evolution equations.

The first published observation of a solitary wave (a single and localized wave) was made by John Scott Russel on the Edinburgh-Glasgow canal in 1834. An account of his observation is given in his 1844 report to the British Association[12]. In this report, Russel describes how he followed a solitary water wave for more than one mile on horse back,

* Corresponding author:

rajan_a100@yahoo.com (Rajan Arora)

Published online at http://journal.sapub.org/am

Copyright (C) 2011 Scientific \& Academic Publishing. All Rights Reserved observing that the wave preserves its original shape. He also noted that higher waves travel faster, that an initial profile evolves into several waves which then move apart and approach solitary waves as time ' $t$ ' tends to infinity, and that solitary waves that move with different speeds, undergo a nonlinear interaction from which they emerge in their original shape.

The Benjamin-Bona-Mahony (BBM) equation has been investigated as a regularized version of the Korteweg-de Vries equation for shallow water waves[11]. It incorporates nonlinear dispersive and dissipative effects[3,4]. In certain theoretical investigations the equation is superior as a model for long waves, and the word "regularized" refers to the fact that, from the standpoint of existence and stability, the equation offers considerable technical advantages over the Korteweg-de Vries equation. In addition to shallow water waves, the equation is applicable to the study of drift waves in plasma or the Rossby waves in rotating fluids. Under certain conditions, it also provides a model of one-dimensional transmitted waves. These find applications in semiconductor devices, optical devices, etc[11]. Apart from these applications, solutions to the BBM equation are interesting in and of themselves.

The aim of this paper is to apply the cosine function-method to obtain the exact solutions for the two different types of nonlinear partial differential equations such as, Benjamin-Bona-Mahony (BBM) equation and Modified Regularized Long Wave (MRLW) equation which are the important soliton equations.

\section{The Cosine-Function Method}

Consider the nonlinear partial differential equation in the form

$$
\phi\left(u_{t}, u_{x}, u^{n} u_{x}, u_{x x x}, u_{x x t}, \ldots\right)=0 \text {, }
$$


where $u(x, t)$ is the solution of (1); $u_{t}$ and $u_{x}$ etc. are the partial derivatives of $u$ with respect to $t$ and $\mathrm{x}$, respectively.

We assume that equation (1) admits travelling wave solution. We use the transformation $u(x, t)=f(\chi)$, where $\chi=x-c t-d, c$ is the speed of the travelling wave and $d$ is a constant. This enables us to use the following changes:

$$
\begin{aligned}
\frac{\partial u}{\partial t}=-c \frac{d f}{d \chi}, \quad \frac{\partial u}{\partial x} & =\frac{d f}{d \chi}, \quad \frac{\partial^{2} u}{\partial x^{2}}=\frac{d^{2} f}{d \chi^{2}}, \quad \frac{\partial^{3} u}{\partial x^{3}} \\
& =\frac{d^{3} f}{d \chi^{3}}, \ldots .
\end{aligned}
$$

Using the above transformation the nonlinear partial differential equation (1) is transformed to nonlinear ordinary differential equation:

$$
\psi\left(f^{\prime}, f^{n} f^{\prime}, f^{\prime \prime \prime}\right)=0 .
$$

By integrating (3) with respect to $\chi$, we obtain

$$
\varphi\left(f, f^{n+1}, f^{\prime \prime}, \ldots\right)=0 .
$$

We write the function $f(\chi)$ in the following form

$$
f(\chi)=\lambda \cos ^{\beta}(\mu \chi),
$$

where $\lambda, \beta$ and $\mu$ are the unknown parameters.

The first derivative of (5) yields

$$
f^{\prime}(\chi)=-\lambda \beta \mu \cos ^{\beta-1}(\mu \chi) \cdot \sin (\mu \chi),
$$

and the second derivative of (5) yields

$$
\begin{gathered}
f^{\prime \prime}(\chi)=\quad-\lambda \mu^{2} \beta \cos ^{\beta}+\lambda \mu^{2} \beta(\beta-1) \cos ^{\beta-2}(\mu \chi) \\
-\lambda \mu^{2} \beta(\beta-1) \cos ^{\beta}(\mu \chi) .
\end{gathered}
$$

We substitute (5) and (6) in (4) to obtain an equation in different powers of cosine functions. Now equating the coefficients of the same powers of cosine functions we obtain a system of algebraic equations in the parameters $\lambda, \beta$ and $\mu$. This system can be solved to obtain the values of $\lambda, \beta$ and $\mu$.

The exact analytical solution of NLPDE (1) is then obtained by substituting the values of the unknown parameters in (5).

\section{Applications}

\subsection{The Benjamin-Bona-Mahony (BBM) Equation}

The Benjamin-Bona-Mahony (BBM) equation is the nonlinear partial differential equation in the following form:

$$
u_{t}+u_{x}+u u_{x}-u_{x x t}=0 \text {, }
$$

where $u(x, t)$ is the solution of (7), $x$ is the space variable and $t$ is the time.

Using the above procedure we obtain

$$
-c \frac{d f}{d \chi}+\frac{d f}{d \chi}+f \frac{d f}{d \chi}+c \frac{d^{3} f}{d \chi^{3}}=0 .
$$

By integrating (8) with respect to $\chi$, we obtain

$$
-c f+f+\frac{f^{2}}{2}+c \frac{d^{2} f}{d \chi^{2}}=0 \text {. }
$$

Now putting the values of $f$ and $d^{2} f / d \chi^{2}$ from (5) and (6) in (9) we obtain

$$
\begin{gathered}
-c \lambda \cos ^{\beta}(\mu \chi)+\lambda \cos ^{\beta}(\mu \chi)+\frac{1}{2} \lambda^{2} \cos ^{2 \beta}(\mu \chi) \\
-c \lambda \mu^{2} \beta \cos ^{\beta}(\mu \chi)+c \lambda \mu^{2} \beta(\beta-1) \cos ^{\beta-2}(\mu \chi) \\
-c \lambda \mu^{2} \beta(\beta-1) \sinh ^{\beta}(\mu \chi)=0 .
\end{gathered}
$$

The equation (10) is satisfied if and only if the following system of algebraic equations holds:

$$
-c \lambda+\lambda-c \lambda \mu^{2} \beta-c \lambda \mu^{2} \beta(\beta-1)=0,
$$

$$
\begin{gathered}
\frac{1}{2} \lambda^{2}+c \lambda \mu^{2} \beta(\beta-1)=0, \\
2 \beta=\beta-2 .
\end{gathered}
$$

Now solving the system (11), we obtain

$$
\begin{gathered}
\beta=-2, \quad \lambda=-3(1-c), \\
\mu= \pm \frac{1}{2} \sqrt{\frac{(1-c)}{c}} .
\end{gathered}
$$

Thus, the exact soliton solution of the BBM equation is

$$
u(x, t)=-3(1-c) \cos ^{-2}\left( \pm \frac{1}{2} \sqrt{\frac{(1-c)}{c}}(x-c t-d)\right) .
$$

\subsection{The Modified Regularized Long Wave (MRLW) Equation}

The modified regularized long wave equation is

$$
u_{t}+u_{x}+u^{2} u_{x}-u_{x x t}=0 \text {, }
$$

where $u(x, t)$ is the solution of (12), $x$ is the space variable and $t$ is the time.

Using the above procedure we obtain:

$$
-c \frac{d f}{d \chi}+\frac{d f}{d \chi}+f^{2} \frac{d f}{d \chi}+c \frac{d^{3} f}{d \chi^{3}}=0 .
$$

By integrating (13) with respect to $\chi$, we obtain

$$
-c f+f+\frac{f^{3}}{3}+c \frac{d^{2} f}{d \chi^{2}}=0 \text {. }
$$

Now putting the values of $f$ and $d^{2} f / d \chi^{2}$ from (5) and (6) in (14) we obtain

$$
\begin{gathered}
-c \lambda \cos ^{\beta}(\mu \chi)+\lambda \cos ^{\beta}(\mu \chi)+\frac{1}{3} \lambda^{3} \cos ^{3 \beta}(\mu \chi) \\
-c \lambda \mu^{2} \beta \cos ^{\beta}(\mu \chi)+c \lambda \mu^{2} \beta(\beta-1) \cos ^{\beta-2}(\mu \chi) \\
-c \lambda \mu^{2} \beta(\beta-1) \cos ^{\beta}(\mu \chi)=0 .
\end{gathered}
$$

The equation (15) is satisfied if and only if the following system of algebraic equations holds:

$$
\begin{gathered}
-c \lambda+\lambda-c \lambda \mu^{2} \beta-c \lambda \mu^{2} \beta(\beta-1)=0, \\
\frac{1}{3} \lambda^{3}+c \lambda \mu^{2} \beta(\beta-1)=0, \\
3 \beta=\beta-2 .
\end{gathered}
$$

Now solving the system (16), we obtain

$$
\begin{gathered}
\beta=-1, \quad \lambda= \pm \sqrt{6(1-c)}, \\
\mu= \pm \sqrt{\frac{(1-c)}{c}} .
\end{gathered}
$$

Thus, the exact soliton solution of the MRLW equation is

$$
u(x, t)= \pm \sqrt{6(1-c)} \cos ^{-1}\left( \pm \sqrt{\frac{(1-c)}{c}}(x-c t-d)\right)
$$

\section{Conclusions}

In this paper, the cosine-function method has been successfully applied to obtain the solution of two nonlinear partial differential equations such as BBM and MRLW equations. The cosine-function method is used to obtain new exact solutions. Thus we can say that the cosine-function method can be extended to solve the system of nonlinear partial differential equations arising in the theory of solitons and other areas.

ACKNOWLEDGEMENTS 
Research funding from the DST, India vide Project grant number SR/FTP/MS-12/2008 is gratefully acknowledged.

\section{REFERENCES}

[1] A. A. Soliman, 2006, The modified extended tanh-function method for solving Burgers type equations, Physica A, 361(2), 394-404

[2] A. H. A. Ali, A. A. Soliman and K. R. Raslan, 2007, Soliton Solution for nonlinear partial differential equations by Cosine-function method, Physics Letters A, 368, 299-304

[3] B. Wang and W. Yang, 1997, Finite-dimensional behaviour for the Benjamin-Bona-Mahony equation, J. Phys. A: Math. Gen., 30, 4877-4885

[4] B. Wang, 1998, Regularity of attractors for the Benjamin-Bona-Mahony equation, J. Phys. A: Math. Gen., 31, 7635-7645

[5] C. T. Yan, 1996, A simple transformation for nonlinear waves, Phys. Lett. A., 224, 77-84

[6] C. Bai, X. Liu and H. Zhao, 2005, New localized excitations in a (2+1)-dimensional Broer-Kaup system, Chin. Phys., 14(2), 0285-0292

[7] D. Kaup, 1975, A higher-order water-wave equation and the method for solving it, Prog. Theor. Phys., 54, 396-408

[8] E. J. Parker and B. R. Duffy, 1996, An automated tanh-function method for finding solitary wave solutions to nonlinear evolution equations, Comput. Phys. Commun., 98, 288-300

[9] E. Fan, 2001, Soliton solutions for a generalized Hirota-Satsuma coupled $\mathrm{KdV}$ equation and a coupled MKdV equation. Phys. Lett. A., 282, 18-22

[10] E. Fan and H. Zhang, 2003, A new algebraic method for finding the line solitons and doubly periodic wave solution to a two-dimensional perturbed kdv equation, Chaos, Solitons and Fractals, 15, 567-574
[11] H. Zhang, G. M. Wei and Y. T. Gao, 2001, On the general form of the BenjaminBona-Mahony equation in fluid mechanics, Czechoslovak Journal of Physics, 51, 373-377

[12] J. S. Russel, Report on waves, Rep. 14th Meet. Brit. Assoc. Adv. Sci., John Murray, York, London, 1844, 311-390

[13] K. Dogan, 2004, An application of the decomposition method for the KdVB equation, Applied Mathematics and Computation, 152(1), 279-288

[14] R. Conte and M. Musette, 1992, Link between solitary waves and projective Riccati equations, Phys. A: Math. Gen., 25(21), 5609-5623

[15] S. A. El-Wakil, S. K. El-Labany, M.A. Zahran and R. Sabry, 2002, Modified extended tanh-function method for solving nonlinear partial differential equations, Phys. Lett. A., 299, 179-188

[16] S. Zhang, B. Wu and S. Lou, 2002, Painleve analysis and special solutions of generalized Broer-Kaup equations, Phys Lett. A., 300, 40-48

[17] W. Hong and Y. Jung, 1999, Auto-backlund transformation and analytic solutions for general variable-coefficient $\mathrm{KdV}$ equation. Phys Lett. A., 257(3), 149-152

[18] Y. Zhang and D. Chen, 2004, Backlund transformation and soliton solutions for the shallow water wave equation. Chaos Solitons and Fractals, 20(2), 343-351

[19] Z. Wang et al., 2005, A method for constructing exact solutions and application to Benjamin Ono equation, Chin. Phys., 14(11), 2158-2163

[20] Abdul Majid Wazwaz, 2009, Partial Differential Equations and Solitary Waves Theory, Springer-Verlag, Heidelberg

[21] G. W. Bluman and S. Kumei, 1989, Symmetries and Differential Equations. Springer-Verlag, Berlin

[22] P. J. Olver, 1993, Applications of Lie Groups to Differential Equations, Springer-Verlag, New York

[23] V. B. Matveev and M. A. Salle, 1991, Darboux Transformation and Solitons, Springer-Verlag, Berlin 